\title{
A VLA SEARCH FOR COSMOLOGICAL H I
}

\author{
Louis Noreau* ${ }^{*} \ddagger$ and Eduardo Hardy ${ }^{* \dagger}$, \\ Département de physique et Observatoire du mont Mégantic \\ Faculté des Sciences et Génie, Université Laval \\ Sainte-Foy (Québec) G1K 7P4 Canada
}

INTRODUCTION: The recent commissioning of the $327 \mathrm{MHz}$ (or $\mathrm{P}$ band) system at the NRAO Very Large Array opens the possibility for a new search for the $21 \mathrm{~cm}$ of H I redshifted to $z \approx 3.3$ and perhaps observing the birth of galaxies as initially suggested by Sunyaev and Zel'dovich $(1972,1975)$. We present and discuss the results of our first observing run in January 1986, and report on the progress on our second run in April 1987.

If QSOs are associated with galaxies, as it is widely believed, then the redshift regime at which they begin to appear may be related to the process of galaxy formation in the early Universe. There is current evidence for a decline in the comoving quasar density beyond $z \approx 3$ (Schmidt $e t$ al. 1987) and some evidence for a cutoff near $z \approx 4$, where very few quasars are known (Osmer 1982, and references therein), which may be associated with the initial epoch of galaxy formation. Past $z \approx 3.5$ all reasonable Friedmann models indicate a slow variation of look-back time with redshift, hence the precise theoretical value of $z$ in this range is less critical when other uncertainties are considered, unless the formation process was extremely peaked in time. Observing the Universe at $z \approx 3.25$ is then of great importance in understanding the genesis and detail of the large scale organization of matter, any detection and measurement of mass there will set fundamental constraints to the later evolution of galaxies and to the amount of presently detected luminous and nonluminous matter.

DIMENSIONS, MASSES, AND DETECTABILITY: Clusters of galaxies have masses in the $10^{14}-10^{15} M_{\mathrm{O}}$ range and sizes $\leq 10 \mathrm{Mpc}$ whereas superclusters, range in mass from $10^{15}$ to $10^{16} M_{0}$, with characteristic dimensions now in the 10 to $100 \mathrm{Mpc}$ interval (Oort 1983). What will be the characteristic sizes of clusters and superclusters at $z \approx 3.25$ ? What fraction of the observable mass will be in the form of $\mathrm{H}$ I and thus susceptible to detection at $327 \mathrm{MHz}$ ?

We adopt as cosmological parameters: $H_{\mathrm{o}}=75 \mathrm{~km} \mathrm{~s}^{-1} \mathrm{Mpc}^{-1}$, and $q_{\mathrm{o}}=0.05$, the luminosity distance is $30,300 \mathrm{Mpc}$, and the scale is 123 " $\mathrm{Mpc}^{-1}$. The local value of the

\footnotetext{
* Guest observers at the NRAO Very Large Array. The National Radio Astronomy Observatory is operated by Associated Universities Inc. under contract with the U. S. National Science Foundation.

$\dagger$ This investigation was supported by NSERC-CANADA.

¥ Supported through a grant from the Fonds F.C.A.R. of Québec to the Mont Mégantic Observatory.
} 
Hubble parameter is $H=370 \mathrm{~km} \mathrm{~s}^{-1} \mathrm{Mpc}^{-1}$. Because of the $(1+z)$ dependence of metric size with redshift due to the expansion one expects a contraction of unbound aggregates of a factor $\sim 4.25$. The cluster size at $z=3.25$ is less than $2.4 \mathrm{Mpc}$, and the superclusters range from 2.4 to $23.5 \mathrm{Mpc}$. This translate into angular sizes of $290^{\prime \prime}$ and $48^{\prime}$. This rarige suggests that the VLA operated in the " $\mathrm{D}$ " configuration is well suited to our purposes since its largest visible structure will be $\sim 40^{\prime}$ and its resolution $\sim 3$ '.

If we consider a supercluster of mass $10^{16} M_{0}$ at $z=3.25$ (a luminosity distance of $\left.D_{L}=4 \times 10^{4} \mathrm{Mpc}\right)$ then if $5 \%$ of the supercluster matter is in $\mathrm{H} \mathrm{I}\left(6 \times 10^{14} M_{\mathrm{O}}\right)$, it follows that a structure extending over $2 \mathrm{Mpc}$ in depth $(780 \mathrm{kHz}$ ) would emit $9 \mathrm{mJy}$ (for $q_{0}=0.05$ ) and $27 \mathrm{mJy}$ (for $q_{0}=0.5$ ). Such sensitivity can be achieved with the VLA at a $3 \sigma$ level with 10 antennas during a 12 hour observation.

JANUARY 1986 OBSERVATIONS: At the time of our first observations, only 10 antennas were fitted with $P$ band receivers. Two fields were surveyed: The first one $(1303+2925)$, near the north galactic pole, contains 7 quasars candidates with $3<z<3.7$. The second field $(1200+2900)$ does not contain any known quasars in the survey redshift range, and was chosen to test the effect of the presence or absence of QSOs. Both fields are far from the galactic plane. A total bandwidth of $6.25 \mathrm{MHz}$ was surveyed.

Internal monochromatic interference at multiples of $0.1,1,5$, and $12.5 \mathrm{MHz}$ was present in the data due to improper shielding of the VLA antenna electronics. The effect of the ringing, or propagation of the emission of the interference signal to other channels, could be reduced considerably by applying "Hanning-smoothing" to the visibilities. The gist of the data reduction was to identify this interference and remove it as much as possible from the data. Fields 1 and 2 were processed in an identical manner; more details on the data reduction can be found in Hardy and Noreau (1987). After elimination of the most damaging interference signal, only $38.6 \%$ and $52.6 \%$ of the original visibilities were kept. The final channel maps were improved by a factor better than 6 compared to the original data.

To eliminate the unsuccessfully removed strong continuum sources and the confusion from the general population of low intensity radio sources, we subtracted from these maps, a general template, produced by averaging our best channel maps. Finally the 32 maps were binned into 8 maps covering $781.4 \mathrm{kHz}$ or $2 \mathrm{Mpc}$ in depth, that is the size of a cluster of galaxy at $z=3.25$. No line signal was detected in these final maps. The noise statistics of these maps appear below:

\begin{tabular}{ccc}
$\begin{array}{c}v \\
\text { MHz }\end{array}$ & $\begin{array}{c}\sigma(\text { Field 1) } \\
\text { mJy/beam }\end{array}$ & $\begin{array}{c}\sigma(\text { Field 2) } \\
\text { mJy/beam }\end{array}$ \\
\hline 331.167 & 15.0 & 19.1 \\
331.948 & 8.5 & 10.4 \\
332.730 & 9.4 & 11.2 \\
333.510 & 15.1 & 17.7 \\
334.292 & 40.7 & 46.0 \\
335.073 & 229.8 & 1421.7 \\
335.854 & 35.2 & 34.1 \\
336.636 & 37.9 & 38.3 \\
\hline Mean* & 23.1 & 25.3 \\
$\sigma_{r m s}$ & 5.4 & 5.3 \\
\hline * excluding the 335.073 map
\end{tabular}


DISCUSSION AND CONCLUSIONS: To analyze our results, we adopt the framework developed by Sunyaev and Zel'dovich (1975, hereafter SZ) to confront our observations with cosmological theory. The total volume surveyed by our January 1986 observations is $7.3 \times 10^{4} \mathrm{Mpc}^{3}$ per field (for $q_{0}=0.05$ ). Considering our very best channels and channel bins with $3 \sigma \approx 30 \mathrm{mJy}$ noise using the results of Table 1 of SZ and scaling the flux density with redshift as $(1+z)^{3}$ we obtain best values for our $\mathrm{H}$ I mass detection limits of $3 \times 10^{14}$ and $1.5 \times 10^{15} M_{0}$ approximately for $q_{0}=0.5$ and 0.05 respectively. Since the first is near the value adopted by SZ as their fiducial mass for single clusters it follows that with our present data we could have detected individual protoclusters only for a closed Universe and only within a restricted number of quiet channels.

Using a present density of clusters of $3 \times 10^{-5} \mathrm{Mpc}^{-3}$ and assuming that protoclusters are in a condition to emit the $\lambda 21 \mathrm{~cm}$ line for $10 \%$ of their age SZ estimate a total of $\mathrm{N} \sim$ $2 \times 10^{5}$ protoclusters in the Universe. For an observing bandwidth $\Delta v$ at $v$ one would expect to detect $N(\Delta v / v)$ objects inside a spherical shell of thickness $\Delta v / v=\Delta z /(1+z)$. Under the present conditions with a bandwidth of $6.25 \mathrm{MHz}$ and a beam surface of $\sim 5$ square degrees (HPBW) the predicted number of intercepted protoclusters is $\sim 0.5$ per field for a combined interception probability of $\sim 1$. If we exclude the frequency bin centered at $335.073 \mathrm{MHz}$ the interception probability is still close to unity and from the mean noise level of $\sim 25 \pm 5(1 \sigma)$ we obtain mean $3 \sigma$ mass sensitivity levels of $\sim(7.5 \pm 1.5) \times 10^{14} M_{0}$ and $(3.8 \pm 0.8) \times 10^{15} M_{0}$ respectively for $q_{o}=0.5$ and 0.05 . These values represent our best estimates of the detection sensitivity of the present experiment within the bandpass used. We were likely to detect a protocluster in a closed Universe and a supercluster (which would occupy most of the bandwidth) even in an open Universe since our detection limit was not far from the mean $\mathrm{H}$ I masses expected. Our search suggests that if the Universe is closed (i.e. $\Omega \geq 1$ ) either the number of protoclusters (in the Sunyaev-Zel'dovich sense) is lower than $\sim 2 \times 10^{5}$ or $z=3.25$ is already too late in the history of the Universe to detect significant amounts of $\mathrm{H}$ I. If quasars are related to superclusters then the limits implied by our survey are even more stringent strongly suggesting that protoclusters had already formed most of their galaxies at an earlier time.

APRIL 1987 OBSERVATIONS: New observations of three fields were secured at the VLA in April 1987. All three selected fields have two or more confirmed quasars in or near the observation bandwidth. Up to 17 antennas were fitted with P-band receiver. A full bandwidth of $12.5 \mathrm{MHz}$ was surveyed. The observation, at the moment of writing, are fully reduced and await analysis. The signal-to-noise ratio should be by at least a factor 4 better than our January 1986 observations.

\section{References}

Hardy E. and Noreau L. (1987) Astron. J. , 94, (in press).

Oort, J. (1983). Ann. Rev. Astron. Astrophys., 21, 373.

Osmer, P. (1982). Astrophys. J., 253, 28.

Schmidt, M., Schneider, D., P., and Gunn, J., E. (1987). Astrophys. J., 316, L1.

Sunyaev, R. A. and Zel'dovich, Ya. B. (1972). Astron. Astrophys., 20, 189.

Sunyaev, R. A. and Zel'dovich, Ya. B. (1975). Mon.Not.R.Astron.Soc. 171, 375. 